research support from: RB has received grants or research support from AbbVie, Merck, and Roche; and has received consultation fees or honoraria for serving as a speaker for AbbVie, Bristol-Myers Squibb, Janssen, Lilly, Merck, Pfizer, and Roche. Jacob Aelion Grant/research support from: JA has received grants or research support from AbbVie, Amgen, AstraZeneca, Bristol-Myers Squibb, Galapagos/Gilead Genentech, GlaxoSmithKline, Lilly, Mallinckrodt, Nektar Therapeutics, Nichi-lko, Novartis, Pfizer, Regeneron, Roche, Sanofi, Selecta Biosciences, and UCB., Gabriela Alperovich Shareholder of: GA is a full-time employee of AbbVie, and may hold AbbVie stock or stock options., Employee of: GA is a full-time employee of AbbVie, and may hold AbbVie stock or stock options., Ying Zhang Shareholder of: $Y Z$ is a former AbbVie employee, and may hold AbbVie stock or stock options., Employee of: $Y Z$ is a former AbbVie employee, and may hold AbbVie stock or stock options., Zailong Wang Shareholder of: ZW is a full-time employee of AbbVie, and may hold AbbVie stock or stock options., Employee of: ZW is a full-time employee of AbbVie, and may hold AbbVie stock or stock options., Ahmed M. Soliman Shareholder of: AS is a full-time employee of AbbVie, and may hold AbbVie stock or stock options., Employee of: AS is a full-time employee of AbbVie, and may hold AbbVie stock or stock options., Ann Eldred Shareholder of: AE is a full-time employee of AbbVie, and may hold AbbVie stock or stock options., Employee of: AE is a full-time employee of AbbVie, and may hold AbbVie stock or stock options., Alan Kivitz Shareholder of: AK is a shareholder of or has received honoraria or fees as a consultant, speaker, or expert witness for AbbVie, Boehringer Ingelheim, Celgene, Flexion, Gilead, GlaxoSmithKline, Janssen, Lilly, Merck, Novartis, Pfizer, Regeneron, Sanofi Genzyme, Sun Pharma, and UCB., Speakers bureau: AK is a shareholder of or has received honoraria or fees as a consultant, speaker, or expert witness for AbbVie, Boehringer Ingelheim, Celgene, Flexion, Gilead, GlaxoSmithKline, Janssen, Lilly, Merck, Novartis, Pfizer, Regeneron, Sanofi Genzyme, Sun Pharma, and UCB., Consultant of: AK is a shareholder of or has received honoraria or fees as a consultant, speaker, or expert witness for AbbVie, Boehringer Ingelheim, Celgene, Flexion, Gilead, GlaxoSmithKline, Janssen, Lilly, Merck, Novartis, Pfizer, Regeneron, Sanofi Genzyme, Sun Pharma, and UCB.

DOI: 10.1136/annrheumdis-2021-eular.2695

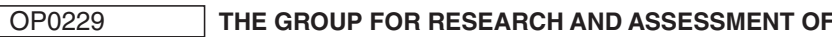 PSORIASIS AND PSORIATIC ARTHRITIS (GRAPPA) TREATMENT RECOMMENDATIONS 2021}

L. C. Coates ${ }^{1}$, E. Soriano ${ }^{2}$, N. Corp ${ }^{3}$, H. Bertheussen ${ }^{4}$, K. Callis-Duffin ${ }^{5}$ C. Barbosa Campanholo ${ }^{6}$, J. Chau $^{7}$, L. Eder ${ }^{8}$, D. Fernandez ${ }^{9}$, O. Fitzgerald ${ }^{10}$, A. Garg ${ }^{11}$, D. D. Gladman ${ }^{12}$, N. Goel ${ }^{13,14}$, S. Grieb ${ }^{15}$, P. Helliwell ${ }^{16}$, M. E. Husni ${ }^{17}$ D. Jadon ${ }^{18}$, A. Katz ${ }^{19}$, D. Laheru ${ }^{20}$, J. Latella ${ }^{21}$, Y. Y. Leung ${ }^{22}$, C. Lindsay ${ }^{23}$, E. Lubrano ${ }^{24}$, L. Mazzuoccolo ${ }^{25}$, R. Mcdonald ${ }^{26}$, P. J. Mease ${ }^{27,28}$, D. O'sullivan ${ }^{29}$ A. Ogdie ${ }^{30}$, W. Olsder ${ }^{31}$, L. Schick ${ }^{15}$, I. Steinkoenig ${ }^{32}$, M. De Wit ${ }^{33}$, D. Van der Windt ${ }^{34}$, A. Kavanaugh ${ }^{35} .{ }^{1}$ University of Oxford, NDORMS, Oxford, United Kingdom; ${ }^{2}$ Hospital Italiano de Buenos Aires, Rheumatology, Buenos Aires, Argentina; ${ }^{3}$ Keele University, School of Primary, Community and Social Care, Keele, United Kingdom; ${ }^{4}$ GRAPPA, Patient Research Partner, Oslo, Norway; ${ }^{5}$ University of Utah, Dermatology, Salt Lake City, United States of America; ${ }^{6}$ Santa Casa de Sao Paulo, Rheumatology, Sao Paulo, Brazil; ${ }^{7}$ GRAPPA, Patient Research Partner, Hong Kong, Hong Kong (SAR); ${ }^{8}$ University of Toronto, Rheumatology, Toronto, Canada; ${ }^{9}$ Hospital Universitario San Ignacio, Rheumatology, Bogota, Colombia; ${ }^{10}$ University College Dublin, Rheumatology, Dublin, Ireland; ${ }^{11}$ Donald and Barbara Zucker School of Medicine at Hofstral Northwell, Dermatology, Hempstead, United States of America; ${ }^{12}$ Toronto Western Hospital, Schroeder Arthritis Institute, Toronto, Canada; ${ }^{13} \mathrm{GRAPPA}$, Patient Research Partner, Durham, United States of America; ${ }^{14}$ Duke University, Rheumatology, Durham, United States of America; ${ }^{15}$ GRAPPA, Patient Research Partner, Seattle, United States of America; ${ }^{16}$ University of Leeds, Leeds Institute of Rheumatology and Musculoskeletal Medicine, Leeds, United Kingdom; ${ }^{17}$ Cleveland Clinic Main Campus, Rheumatology, Cleveland, United States of America; ${ }^{18}$ University of Cambridge, Rheumatology, Cambridge, United Kingdom; ${ }^{19} \mathrm{GRAPPA}$, Patient Research Partner, Haifa, Israel; ${ }^{20} \mathrm{Ch}$ urchill Hospital, Dermatology, Oxford, United Kingdom; ${ }^{21}$ GRAPPA, Patient Research Partner, Connecticut, United States of America; ${ }^{22}$ Singapore General Hospital, Rheumatology, Singapore, Singapore; ${ }^{23}$ GRAPPA, Patient Research Partner, Propser, United States of America; ${ }^{24}$ University of Molise, Rheumatology, Campobasso, Italy; ${ }^{25}$ Hospital Italiano de Buenos Aires, Dermatology, Buenos Aires, Argentina; ${ }^{26}$ GRAPPA, Patient Research Partner, Toronto, Canada; ${ }^{27}$ Swedish Medical Center, Rheumatology, Seattle, United States of America; ${ }^{28}$ University of Washington, Rheumatology, Seattle, United States of America; ${ }^{29}$ GRAPPA, Patient Research Partner, Dublin, Ireland; ${ }^{30}$ University of Pennsylvania, Rheumatology, Philadelphia, United States of America; ${ }^{31}$ GRAPPA, Patient Research Partner, Eindhoven, Netherlands; ${ }^{32}$ GRAPPA, Patient Research Partner, Cleveland, United States of America; ${ }^{33}$ GRAPPA, Patient Research Partner, Amsterdam, Netherlands; ${ }^{34}$ Keele University, School of Primary Community and Social Care, Keele, United Kingdom; ${ }^{35}$ University of California San Diego, Rheumatology, La Jolla, United States of America
Background: Since the 2015 GRAPPA treatment recommendations were published, therapeutic options and management strategies for psoriatic arthritis (PsA) have advanced considerably.

Objectives: The goal of the GRAPPA recommendations update is to develop high quality, evidence-based recommendations for the treatment of PsA, including related conditions and comorbidities.

Methods: GRAPPA rheumatologists, dermatologists and patient research partners (PRPs) updated overarching principles for the management of adults with PsA by consensus. Principles considering use of biosimilars and tapering/discontinuing of therapy were added to this update. Systematic literature searches based on data publicly available from three databases (MEDLINE, EMBASE, and Cochrane CENTRAL) were conducted from the end of the previous recom mendations' searches through August 2020. Additional abstract searches were performed for conference presentations in 2017-2020. Searches covered PsA treatments (peripheral arthritis, axial arthritis, enthesitis, dactylitis, skin, and nail disease). Additional searches were performed for related conditions (uveitis and IBD) and comorbidities evaluating their impact on safety and treatment outcomes. Individual groups assessed the risk of bias and applied the GRADE system to generate strong or conditional recommendations for therapies within the domain groups and for the management of comorbidities and related conditions. These recommendations were then incorporated into an overall treatment schema.

Results: Updated, evidence-based treatment recommendations are shown (Table 1). Since 2015, many new medications have been incorporated. Additiona results for older medications, such as methotrexate, have been published across PsA domains. Based on the evidence, the treatment recommendations developed by individual groups were incorporated into the overall schema including principles for management of arthritis, spondylitis, enthesitis, dactylitis, skin, and nail disease in PsA, and associated conditions (Figure 1). Choice of therapy for an individual should ideally address all of the domains that impact on that patient, supporting shared decision making with the patient involved. Additional consideration in the recommendations was given to key associated conditions and comorbidities as these often impact on therapy choice.

Conclusion: These GRAPPA treatment recommendations provide up to date, evidence-based guidance to providers who manage and treat adult patients with PSA These recommendations are based on domain-based strategy for PsA and supplemented by overarching principles developed by consensus of GRAPPA members.

\begin{tabular}{|c|c|c|c|c|c|}
\hline Indication & $\begin{array}{l}\text { Strong } \\
\text { For }\end{array}$ & $\begin{array}{l}\text { Conditional } \\
\text { For }\end{array}$ & $\begin{array}{l}\text { Conditional } \\
\text { Against }\end{array}$ & $\begin{array}{c}\text { Strong } \\
\text { Against }\end{array}$ & $\begin{array}{l}\text { Insufficient } \\
\text { evidence }\end{array}$ \\
\hline $\begin{array}{l}\text { Peripheral } \\
\text { Arthritis } \\
\text { DMARD } \\
\text { Naïve }\end{array}$ & $\begin{array}{l}\text { csDMARDs, } \\
\text { TNFi, PDE4i, } \\
\text { IL-12/23i, IL-17i, } \\
\text { IL-23i, JAKi }\end{array}$ & $\begin{array}{l}\text { NSAIDs, } \\
\text { oral CS, IA CS, }\end{array}$ & IL-6i, & & \\
\hline $\begin{array}{l}\text { Peripheral } \\
\text { Arthritis } \\
\text { DMARD } \\
\text { IR }\end{array}$ & $\begin{array}{l}\text { TNFi, IL-12/23i, } \\
\text { IL-17i, IL-23i, } \\
\text { JAKi }\end{array}$ & $\begin{array}{l}\text { PDE4i, other } \\
\text { csDMARD, } \\
\text { NSAIDs, oral } \\
\text { CS, IA CS, }\end{array}$ & IL-6i, & & \\
\hline $\begin{array}{l}\text { Peripheral } \\
\text { Arthritis }\end{array}$ & $\begin{array}{l}\text { TNFi, IL-17i, } \\
\text { IL-23i, JAKi, }\end{array}$ & $\begin{array}{l}\text { NSAIDs, oral } \\
\text { CS, IA CS, } \\
\text { IL-12/23i, PDE4i, }\end{array}$ & IL-6i, & & \\
\hline bDMARD IR & & CTLA-4-Ig & & & \\
\hline $\begin{array}{l}\text { Axial } \\
\text { arthritis, } \\
\text { Biologic } \\
\text { Naïve }\end{array}$ & $\begin{array}{l}\text { NSAIDs, } \\
\text { Physiotherapy, } \\
\text { simple analgesia, } \\
\text { TNFi, IL-17i, JAKi }\end{array}$ & $\begin{array}{l}\text { CS SIJ injections, } \\
\text { bisphosphonates }\end{array}$ & & $\begin{array}{l}\text { Cs } \\
\text { DMARDs, } \\
\text { IL-6i, }\end{array}$ & IL-12/23i, IL-23i \\
\hline $\begin{array}{l}\text { Axial PsA, } \\
\text { Biologic } \\
\text { IR }\end{array}$ & $\begin{array}{l}\text { NSAIDs, } \\
\text { Physiotherapy, sim- } \\
\text { ple analgesia, TNFi, } \\
\text { IL-17i, JAKi }\end{array}$ & & & $\begin{array}{l}\text { csD- } \\
\text { MARDs, } \\
\text { IL-6i, }\end{array}$ & IL-12/23i, IL-23i \\
\hline Enthesitis & $\begin{array}{l}\text { TNFi, IL-12/23i, } \\
\text { IL-17i, PDE4i, } \\
\text { IL-23i, JAKi }\end{array}$ & $\begin{array}{l}\text { NSAIDs, phys- } \\
\text { iotherapy, CS } \\
\text { injections, MTX }\end{array}$ & & IL-6i, & $\begin{array}{l}\text { Other cs } \\
\text { DMARDs }\end{array}$ \\
\hline Dactylitis & $\begin{array}{l}\text { TNFi IL-12/23i, } \\
\text { IL-17i, IL-23i, } \\
\text { JAKi, PDE4i }\end{array}$ & $\begin{array}{l}\text { NSAIDs, CS } \\
\text { injections, MTX }\end{array}$ & $\begin{array}{l}\text { Other } \\
\text { csDMARDs }\end{array}$ & & \\
\hline Psoriasis & $\begin{array}{l}\text { Topicals, photother- } \\
\text { apy, csDMARDs, } \\
\text { TNFi, IL-12/23i, } \\
\text { IL-17i, IL-23i, } \\
\text { PDE4i, JAKi }\end{array}$ & Acitretin & & & \\
\hline $\begin{array}{l}\text { Nail } \\
\quad \text { psoriasis }\end{array}$ & $\begin{array}{l}\text { TNFi, IL12/23i, } \\
\text { IL17i, IL23i, } \\
\text { PDE4i }\end{array}$ & $\begin{array}{l}\text { Topical CS, } \\
\text { tacrolimus and } \\
\text { calcipotriol } \\
\text { combination or } \\
\text { individual ther- } \\
\text { apies, Pulsed } \\
\text { dye laser, } \\
\text { csDMARDs, } \\
\text { acitretin, JAKi }\end{array}$ & & & $\begin{array}{l}\text { Topical } \\
\text { Cyclosporine } \\
\text { /Tazarotene, } \\
\text { Fumarate, } \\
\text { Fumaric Acid } \\
\text { Esters, UVA } \\
\text { and UVB } \\
\text { Phototherapy, } \\
\text { Alitretinoin }\end{array}$ \\
\hline IBD & $\begin{array}{l}\text { TNFi (not ETN), } \\
\text { IL-12/23i, JAKi }\end{array}$ & & & IL-17i & \\
\hline Uveitis & TNFi (not ETN) & & & & \\
\hline
\end{tabular}




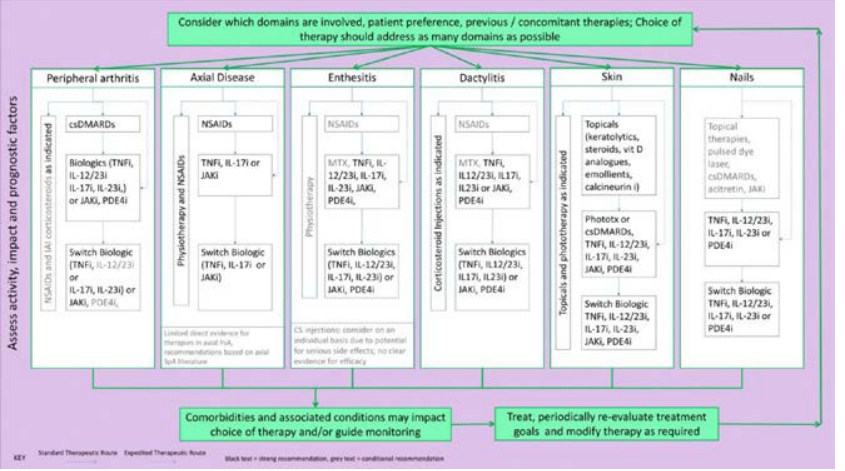

Disclosure of Interests: Laura C Coates Speakers bureau: AbbVie, Amgen, Biogen, Celgene, Gilead, Eli Lilly, Janssen, Medac, Novartis, Pfizer, and UCB, Consultant of: AbbVie, Amgen, Boehringer Ingelheim, Bristol-Myers Squibb, Celgene, Eli Lilly, Gilead, Janssen, Novartis, Pfizer, and UCB, Grant/research support from: AbbVie, Amgen, Celgene, Eli Lilly, Pfizer, and Novartis, Enrique Soriano Speakers bureau: AbbVie, Amgen, Bristol-Myers Squibb,GSK, Genzyme, Janssen, Lilly, Novartis, Pfizer, Roche, Sandoz, Sanofi, UCB, Consultant of: AbbVie, Amgen, Bristol-Myers Squibb,GSK, Genzyme, Janssen, Lilly, Novartis, Pfizer, Roche, Sandoz, Sanofi, UCB, Grant/research support from: AbbVie, Janssen, Novartis Pharma, Pfizer, Roche, and UCB, Nadia Corp: None declared, Heidi Bertheussen Consultant of: Pfizer, Kristina Callis-Duffin Consultant of: AbbVie, Amgen, Bristol-Myers Squibb, Celgene, Lilly, Janssen, Novartis, Pfizer, Sienna Biopharmaceuticals, Stiefel Laboratories, UCB, Ortho Dermatologics, Inc, Regeneron Pharmaceuticals, Inc., Anaptys Bio, Boehringer Ingelheim., Cristiano Barbosa Campanholo Speakers bureau: AbbVie, Eli Lilly, Janssen, Novartis, Pfizer, and UCB, Consultant of: AbbVie, Bristol-Myers Squibb, Eli Lilly, Janssen, Novartis, Pfizer, and UCB, Jeffrey Chau: None declared, Lihi Eder Consultant of: Abbvie, UCB, Janssen, Eli Lily, Pfizer, Novartis, Grant/research support from: Abbvie, UCB, Janssen, Eli Lily, Pfizer, Novartis, Daniel Fernandez Consultant of: Abbvie, UCB, Roche, Janssen, Pfizer, Amgen and Brystol, Grant/research support from: Abbvie, UCB, Roche, Janssen, Pfizer, Amgen and Brystol, Oliver FitzGerald Speakers bureau: AbbVie, Janssen and Pfizer Inc, Consultant of: BMS, Celgene, Eli Lilly, Janssen and Pfizer Inc, Grant/research support from: AbbVie, BMS, Eli Lilly, Novartis and Pfizer Inc, Amit Garg Consultant of: Abbvie, Amgen, Asana Biosciences, Bristol Myers Squibb, Boehringer Ingelheim, Incyte, InflaRx, Janssen, Pfizer, UCB, Viela Biosciences, Grant/research support from: Abbvie, Dafna D Gladman Consultant of: Abbvie, Amgen, BMS, Eli Lilly, Galapagos, Gilead, Jansen, Novartis, Pfizer and UCB, Grant/research support from: Abbvie, Amgen, Eli Lilly, Jansen, Novartis, Pfizer and UCB, Niti Goel: None declared, Suzanne Grieb: None declared, Philip Helliwell Speakers bureau: Janssen, Novartis, Pfizer, Consultant of: Eli Lilly, M Elaine Husni Consultant of: Abbvie, Amgen, Janssen, Novartis, Lilly, UCB, Regeneron, and Pfizer, Deepak Jadon Speakers bureau: AbbVie, Amgen, Celgene, Eli Lilly, Gilead, Healthcare Celltrion, Janssen, MSD, Novartis, Pfizer, Roche, Sandoz, UCB, Consultant of: AbbVie, Amgen, Celgene, Eli Lilly, Gilead, Healthcare Celltrion, Janssen, MSD, Novartis, Pfizer, Roche, Sandoz, UCB, Grant/research support from: AbbVie, Amgen, Celgene, Eli Lilly, Gilead, Healthcare Celltrion, Janssen, MSD, Novartis, Pfizer, Roche, Sandoz, UCB, Arnon Katz: None declared, Dhruvkumar Laheru: None declared, John Latella: None declared, Ying Ying Leung Speakers bureau: Novartis, AbbVie, Eli Lilly, Janssen, Consultant of: Pfizer and Boehringer Ingelheim, Grant/research support from: Pfizer and conference support from AbbVie, Christine Lindsay Shareholder of: Amgen, Employee of: Aurinia pharmaceuticals, Ennio Lubrano Speakers bureau: Alfa-Sigma, Abbvie, Galapagos, Janssen Cilag, Lilly., Consultant of: Alfa-Sigma, Abbvie, Galapagos, Janssen Cilag, Lilly., Luis Mazzuoccolo Speakers bureau: Abbvie, Amgen, Novartis, Elli Lilly, Jansen, Consultant of: Abbvie, Amgen, Novartis, Elli Lilly, Jansen, Roland McDonald: None declared, Philip J Mease Speakers bureau: AbbVie, Amgen, Eli Lilly, Janssen, Novartis, Pfizer and UCB, Consultant of: AbbVie, Amgen, Boehringer Ingelheim, Bristol-Myers Squibb, Eli Lilly, Galapagos, Gilead Sciences, GlaxoSmithKline, Janssen, Novartis, Pfizer, SUN and UCB, Grant/research support from: AbbVie, Amgen, Bristol-Myers Squibb, Celgene, Eli Lilly, Galapagos, Gilead Sciences, Janssen, Novartis, Pfizer, SUN and UCB, Denis O'Sullivan: None declared, Alexis Ogdie Consultant of: AbbVie, Amgen, BMS, Celgene, Corrona, Gilead, Janssen, Lilly, Novartis, and Pfizer, Grant/research support from: Novartis and Pfizer and Amgen, Wendy Olsder: None declared, Lori Schick: None declared, Ingrid Steinkoenig: None declared, Maarten de Wit Consultant of: AbbVie, BMS, Celgene, Janssen, Lilly, Novartis, Pfizer, Roche, Danielle van der Windt: None declared, Arthur Kavanaugh Speakers bureau: AbbVie, Amgen, BMS, Eli Lilly, Gilead Janssen, Novartis, Pfizer, UCB, Consultant of: AbbVie, Amgen, BMS, Eli Lilly, Gilead Janssen, Novartis, Pfizer, UCB DOI: 10.1136/annrheumdis-2021-eular.4091

\section{OP0230 EFFICACY AND SAFETY OF GUSELKUMAB IN PATIENTS WITH ACTIVE PSORIATIC ARTHRITIS WHO DEMONSTRATED INADEQUATE RESPONSE TO TUMOR NECROSIS FACTOR INHIBITION: WEEK 24 RESULTS OF A PHASE 3B, RANDOMIZED, CONTROLLED STUDY}

L. C. Coates ${ }^{1}$, L. Gossec ${ }^{2}$, E. Theander ${ }^{3}$, P. Bergmans ${ }^{4}$, M. Neuhold ${ }^{5}$, C. Karyekar ${ }^{6}$, M. Shawi ${ }^{6}$, W. Noel ${ }^{5}$, G. Schett ${ }^{7}$, I. Mcinnes ${ }^{8} .{ }^{1}$ University of Oxford, Nuffield Department of Orthopaedics, Rheumatology and Musculoskeletal Sciences, Oxford, United Kingdom; ${ }^{2}$ Sorbonne Université, Professor of Rheumatology, Paris, France; ${ }^{3}$ Janssen Scientific Affairs, LLC, Immunology, Solna, Sweden; ${ }^{4}$ Janssen, Biostatistics, Breda, Netherlands; ${ }^{5} J a n s s e n$ Scientific Affairs, LLC, Immunology, Brussels, Belgium; ${ }^{6}$ Janssen Global Services, LLC, Immunology, Horsham, United States of America; ${ }^{7}$ University of Erlangen-Nuremberg, Internal Medicine 3, Erlangen, Germany; ${ }^{8}$ University of Glasgow, Institute of Infection, Immunity and Inflammation, Glasgow, United Kingdom

Background: Guselkumab (GUS), a selective monoclonal antibody targeting the interleukin-23p19 subunit, has demonstrated efficacy in 2 pivotal Ph3 psoriatic arthritis (PsA) studies (DISCOVER- $1,{ }^{1}$ DISCOVER- ${ }^{2}$ ).

Objectives: Evaluate GUS efficacy and safety in PsA patients (pts) with inadequate response (IR) to tumor-necrosis-factor inhibition (TNFi) through Week24 (W24) of the Ph3b COSMOS study.

Methods: In this randomized, double-blind, placebo (PBO)-controlled trial, 285 pts with active PsA ( $\geq 3$ swollen \& $\geq 3$ tender joints) who demonstrated lack of benefit or intolerance to 1-2 TNFi were randomized 2:1 to subcutaneous GUS $100 \mathrm{mg}(\mathrm{n}=189)$ or PBO $(n=96)$ at W0, W4, then every 8 weeks (Q8W) through W44 (with PBO crossover to GUS at W24). At W16, pts who met early escape (EE) criteria ( $<5 \%$ improvement in both tender \& swollen joint counts) also could switch from PBO to GUS. The primary efficacy endpoint was ACR20 response at W24 among randomized, treated pts. Pts missing ACR20 data at W24 or who met treatment failure criteria (including meeting EE criteria at W16) were considered nonresponders (NRs). Subgroup analyses were performed to assess consistency of primary treatment effect based on demographics, disease characteristics, and medication use at baseline. Prespecified sensitivity analyses included 'Per-Protocol' (PP) (excluded pts with major protocol deviations) and 'EE-Correction' (included pts incorrectly routed to EE) analyses. Adverse events (AEs) were summarized by treatment received.

Results: Baseline characteristics were similar across GUS and PBO pts, though a higher proportion of females and more severe joint symptoms were seen in the GUS group. At W24, $44.4 \%$ of GUS vs $19.8 \%$ of PBO pts achieved ACR20 $(p<0.001)$ (Figure). GUS was superior to PBO for all major secondary endpoints. Efficacy was consistent across subgroups defined by baseline characteristics, including in pts who discontinued prior TNFi use due to inadequate efficacy ( $84 \%$ GUS vs $81 \%$ PBO) and safety ( $16 \%$ GUS vs $19 \%$ PBO) (Table). 20 pts (12 GUS, 8 PBO) were incorrectly routed to EE. Results of PP (48.8\% vs $23.8 \%)$ and $\mathrm{EE}$-correction $(48.1 \%$ vs $19.8 \%)$ sensitivity analyses were consistent with the primary analysis (Figure). AEs were similar between GUS- and PBO-treated pts (Table).

Table 1. Baseline characteristics of, and adverse events reported by, randomized and treated COSMOS pts

\begin{tabular}{lcc}
\hline & $\begin{array}{c}\text { GUS 100 } \mathbf{~ m g ~ Q 8 W ~} \\
\text { (N=189) }\end{array}$ & $\begin{array}{c}\text { PBO } \\
\text { (N=96) }\end{array}$ \\
\hline Age, y & 49 & 49 \\
Sex, Female & $54 \%$ & $46 \%$ \\
Duration of PsA, y & 8.3 & 8.7 \\
Body mass index, kg/m & 29 & $31^{\mathrm{a}}$ \\
Swollen (0-66) / tender (0-68) joint count & $10 / 21$ & $9 / 18$ \\
Pt pain / Pt global arthritis / Physician global disease, & $6.5 / 6.5 / 6.9$ & $6.0 / 6.2$ \\
0-10 cm VAS & & 16.4 \\
Health Assessment Questionnaire-Disability Index, 0-3 & $1.3^{\mathrm{b}}$ & 1.2 \\
C-reactive protein, mg/dL & $1.2^{\mathrm{b}}$ & 1.2 \\
Methotrexate use at baseline & $56 \%$ & $53 \%$ \\
Psoriatic body surface area, \% & 17.9 & 13.4 \\
Number of prior TNFi: 1 / & $88 \% / 12 \%$ & $89 \% / 11 \%$ \\
Reason for prior TNFi discontinuation: Efficacy / Safety & $84 \% / 16 \%{ }^{*}$ & $81 \% / 19 \%$ \\
Pts with $\geq 1$ AE / SAE & $37 \% / 3 \%$ & $48 \% / 3 \%$ \\
Pts with $\geq 1$ infection / serious infection & $18 \% / 0 \%$ & $20 \% / 0 \%$ \\
Pts with $\geq 1$ AE leading to study agent & $2 \%$ & $2 \%$ \\
discontinuation & & \\
Pts with $\geq 1$ malignancy & $0.4 \%$ & 0 \\
Pts with $\geq 1$ injection-site reaction & $2 \%$ & $1 \%$ \\
\hline
\end{tabular}

Data shown are mean or \%. ${ }^{a} \mathrm{~N}=95 ;{ }^{\mathrm{b}} \mathrm{N}=188$. *Missing for $1 \mathrm{pt} . \mathrm{SAE}$ - serious adverse events; VAS - visual analog scale

Conclusion: In this Ph3b, placebo-controlled study of PsA pts with IR to 1-2 TNFi, GUS $100 \mathrm{mg}$ Q8W elicited a significantly higher ACR20 response rate vs. 